\title{
The mediator role of stigma in the association of mindfulness and social engagement among breast cancer survivors in China
}

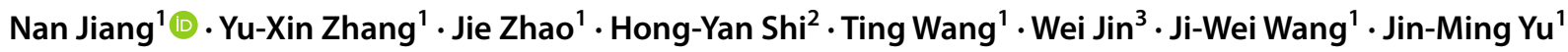

Received: 19 October 2021 / Accepted: 28 January 2022 / Published online: 22 February 2022

(c) The Author(s), under exclusive licence to Springer-Verlag GmbH Germany, part of Springer Nature 2022

\begin{abstract}
Purpose This study aims to explore the association between mindfulness and social engagement among Chinese breast cancer survivors (BCSs) and the mediator role of stigma in the relation of mindfulness and social engagement.

Methods This cross-sectional study was conducted among 937 BCSs from March to April 2021 in Shanghai, China. Data were collected using the Mindful Attention Awareness Scale, the Stigma Scale for Chronic Illness 8-item version, and the index of social engagement. Descriptive statistics, independent-sample $t$-test, one-way ANOVA, and regression analyses were used to explore the role of stigma in the association of mindfulness and social engagement among Chinese BCSs.

Results Social engagement levels differed significantly by participant's BMI, education level, employment status, personal monthly income, monthly per capita household income. Mindfulness was positively correlated with social engagement, and stigma was negatively correlated with mindfulness and social engagement among Chinese BCSs. Stigma plays a complete mediating role in the relationship between mindfulness and social engagement in BCSs.

Conclusion In the practice of individual mindfulness intervention on social engagement of BCSs, health care providers should identify and eliminate the constraints, which restrain the reduction of stigma level while individual mindfulness is being enhanced.
\end{abstract}

Keywords Breast cancer survivors $\cdot$ Mindfulness $\cdot$ Stigma $\cdot$ Social engagement

\section{Introduction}

Global cancer statistics 2020 shows that breast cancer is the most commonly diagnosed cancer in women worldwide [1]. The improvement of early diagnosis and treatment has led to a steady rise in long-term BCSs [2]. However, after treatment ends for breast cancer, BCSs experience changes in functional capacity, resulting in a variety of social and

\footnotetext{
Wei Jin

jsenyao@126.com

$\triangle$ Ji-Wei Wang

jiweiwang@fudan.edu.cn

Nan Jiang

16301020008@fudan.edu.cn

Yu-Xin Zhang

15301020051@fudan.edu.cn

Jie Zhao

19211020034@fudan.edu.cn

Hong-Yan Shi

86259225@qq.com
}

psychological problems such as adjustment disorders, anxiety, depression, and emotional distress [3], which hinder the performance of their daily activities and social engagement [4]. Social engagement refers to the maintenance of social connections and participation in social activities [5], including actively contacting with relatives or friends, participating in recreational social activities, etc., which is considered a higher level of health needs [6]. For BCSs, it

Ting Wang

21211020133@m.fudan.edu.cn

Jin-Ming Yu

jmy@fudan.edu.cn

1 Key Lab of Health Technology Assessment of Ministry of Health, Department of Preventive Medicine and Health Education, School of Public Health, Fudan University, 130 Dong-An Road, Shanghai 200032, China

2 Dental Disease Prevention and Treatment Center of Minhang District, 1038 Fanxing Road, Shanghai 201107, China

3 Department of Health Instruction, Shanghai Municipal Center for Health Promotion, Shanghai 200040, China 
is of significant health benefits to participate in community activities, including decreased stress and anxiety, improved self-esteem and physical capacity, and facilitated recovery of BCSs $[7,8]$. Studies have shown that improving emotional regulation [9], self-efficacy, and social support [10] can increase the social engagement of BCSs.

Recent studies have shown that stigma has a significant impact on social engagement [11]. Corrigan and colleagues distinguished stigma in felt/perceived stigma (the awareness of the discriminatory stereotype around the own illness), enacted stigma (being discriminated against or treated unfairly by others in actual encounters), and internalized/ self-stigma (internalization and acceptance of the discriminatory stereotype) $[12,13]$. When stigma is internalized, the negative perception can lead to negative emotions and attitudes such as shame, guilt, depression, and fear of discrimination and the disease itself [14]. BCSs with stigma may accept negative societal evaluations, further negatively evaluating themselves as inferior to others $[15,16]$. Importantly, the stigma of cancer survivors can lead to rejection of others' favor, avoidance of communication with others, and withdrawal from participation in social activities [17, 18]. Moreover, a study indicated that the stigma not only delays women's early engagement in caring but also hinders women from remaining engaged with care through to treatment completion [19]. There is empirical evidence that three types of stigma are prevalent among BCSs [20, 21], negatively impacting social engagement [11]. Hence, interventions are needed to reduce the stigma of BCSs. Regarding "perceived and enacted stigma," various forms of health education for the public and intergroup contact could eradicate prejudices and misconceptions about breast cancer and reduce stigma [22]. And for individuals' "internalized stigma," improving mindfulness, increasing self-compassion, and promoting individual self-esteem are considered to be effective approaches [23-25].

Mindfulness means paying attention in a particular way: on purpose, in the present moment, and nonjudgmentally [26]. Mindfulness could reduce the level of stigma by effectively increasing positive reappraisal and reducing negative rumination [27], and their negative relationship has been demonstrated in multiple studies [27-30]. In addition, studies have indicated that mindfulness can increase interpersonal skills, improve social cognition, and promote participation in social activities [31]. One of the mediating mechanisms by which mindfulness works is that it can affect an individual's behavior by regulating an individual's emotions [32]. For example, mindfulness has a positive impact on work engagement by increasing positive effects and optimism [33]. By reducing awareness of their negative thoughts, body sensations, and emotions and maintaining a calm and open mind toward the present moment, mindfulness has been shown to have benefits for emotion regulation in BCSs [30, 34], and thus influencing individuals' social interactions and social behaviors [35].

In a society with a large number of BCSs, it is important to identify the impacts of mindfulness on social engagement and to find out the potential intervention approaches. According to the literature, an explanation of the role of stigma in the association of mindfulness and social engagement among BCSs is lacking. Therefore, this study aimed to explore the association between mindfulness and social engagement among Chinese BCSs, and the mediator role of stigma in the relation of mindfulness and social engagement. Based on the above point of view, we hypothesized that mindfulness exerts an effect on individuals' social engagement behavior by influencing stigma.

\section{Methods}

\section{Participants}

In this study, participants were recruited from registered members of the Shanghai Cancer Rehabilitation Club (SCRC) from March to April 2021 using a convenience sampling method. SCRC has a three-level management network: the city, the district, and the street. Members of SCRC come from communities in 16 districts of Shanghai. There is a total of about 50,000 BCSs in Shanghai and about 5000 registered BCSs in SCRC. Before the participants were recruited, we calculate the sample size of the mediation model by using an application based on Monte Carlo Power Analysis Simulation, which was developed by Schoemann, Boulton, and Short [36]. The result showed that a sample size required for 0.9 power is at least 680 . Recruitment invitations were sent to sixteen district-level SCRCs. Four districts were recruited on a first-come, first-served basis. Finally, all BCSs registered with the SCRC in these four districts, a total of 1496 BCSs from $41 \mathrm{WeChat}$ groups (one for each street or town), were recruited. All BCSs in SCRC can access recruitment information through the WeChat groups. Other BCSs in the four districts who were not recruited either did not meet the inclusion criteria, either did not attach importance to the recruitment information, or believed that the study did not have tangible benefits for them, and did not want to participate. The inclusion criteria for this study including (1) women over the age of 18 years; (2) ductal carcinoma in situ (DCIS) or stage I-III breast cancer; (3) breast cancer treatment completed more than 3 months with no recurrence; and (4) be able to independently participate in cancer club activities. Exclusion criteria include (1) conditions that would interfere with questionnaire completion (e.g., chemo brain, dementia, cognitive impairment, etc.).

The questionnaire was sent to each participant via the WeChat group, which means the data of this study was 
collected through an online survey. Three quality control questions were added to the questionnaire to verify the quality of the questionnaire completion, for example: please choose B for this question. Questionnaires with correct answers to all three quality control questions were considered valid. In addition, the questions that each participant needs to fill in were set as mandatory, which means that participants would not be able to submit questionnaires if they did not complete these questions. So, there are no missing values in the collected data. For each participant's WeChat account, we limited the questionnaire to be filled out only once. Therefore, no participant will submit the questionnaire repeatedly. Finally, 1012 questionnaires were collected and the response rate was $84.33 \%$. Among them, 937 questionnaires were valid, and the valid rate was $92.59 \%$. Informed consent was obtained from each participant in the study. The study was approved by the Medical Research Ethics Committee of the School of Public Health, Fudan University (the international registry no. IRB00002408 and FWA00002399).

\section{Measurement}

Data were collected through a four-part survey, including demographic and clinical characteristics, mindfulness, stigma, and social engagement. The researchers calculated mean scores for the multi-item measures, thus keeping the 1-6 scoring for mindfulness and the 1-5 scoring for stigma and social engagement.

\section{Mindfulness}

The Mindful Attention Awareness Scale (MAAS) consists of 15 items, designed to measure participants' mindfulness [37]. Each item was scored on a 6-point scale from 1 (almost always) to 6 (almost never). Mean scores ranged from 1 to 6 , with a higher score indicating a higher level of mindfulness. The Chinese version of MAAS has adequate scale reliability [38]. For our study, Cronbach's alpha was 0.89 , implying good internal consistency reliability.

\section{Stigma}

The Stigma Scale for Chronic Illness 8-item version (SSCI8 ) is a brief self-assessment tool to measure the perceived level of stigma [39]. SSCI-8 is an 8-item newly developed short-form instrument that scored on a 5-point Likert scale from 1 (never) to 5 (always). Mean scores ranged from 1 to 5 , the higher the score the more stigma was perceived. However, SSCI- 8 has not yet been used in the Chinese breast cancer population. After obtaining permission to translate and use the questionnaire, the research team translated the questionnaire into Chinese. For our study, Cronbach's alpha of the SSCI-8 Chinese version was 0.90, implying acceptable internal consistency reliability.

\section{Social engagement}

Social engagement was measured using the 6-item index of social engagement (ISE), which was developed to measure the social engagement of older adults in nursing homes in 1995 [40]. In our study, the ISE was adjusted scored on a 5-point Likert scale from 1 (never) to 5 (always). Mean scores ranged from 1 to 5 ; higher scores indicate higher levels of social engagement. However, ISE has not yet been used in the Chinese breast cancer population. After obtaining permission to translate and use the questionnaire, the research team translated the questionnaire into Chinese. For our study, Cronbach's alpha of the ISE Chinese version was 0.84 , indicating acceptable internal consistency reliability.

\section{Translation}

After obtaining permission to translate and use the questionnaire, our research team sinicized the SSCI- 8 and ISE, following these steps: (1) The original questionnaire was independently translated into simplified Chinese by two native Chinese-speaking experts. Then, a third Chinese-speaking expert coordinated their translations and combined them as the initial Chinese version. (2) A native English-speaking expert, who does not know the original English version of the questionnaire, back-translated the initial Chinese version into English. (3) Finally, researchers invited three more native Chinese-speaking experts to check the translated and back-translated versions to select the most appropriate translation for each item. And if the provided translations were unacceptable, alternative translations that were more suitable for the Chinese context will be provided. (4) In the absence of substantial differences, the Chinese version was considered final. The translation was finalized after the project members performed a final quality review again.

\section{Statistical analysis}

The software STATA 15.1SE (STATA Corp, College Station, TX, USA, http://www.stata.com) for Windows was used for data analysis. Descriptive statistics calculated sample characteristics (age, body mass index (BMI; World Health Organization classification), marital status, education, employment status, personal monthly income (CNY¥; all income are presented in Chinese yuan), monthly per capita household income (CNY¥), duration of disease, surgery type, comorbidity). For every scale, Cronbach's alpha was 
calculated to measure the internal consistency. Use $t$-test and ANOVA to analyze differences in social engagement. Pearson correlation analysis was performed to assess correlations among mindfulness, stigma, and social engagement.

The mediation models were examined using the following steps suggested by Baron and Kenny [41]: (1) in the first equation, social engagement was regressed on mindfulness; (2) in the second equation, stigma was regressed on mindfulness; (3) in the third equation, social engagement was regressed on mindfulness and stigma. When the coefficient of the first step and the second step is significant, and the coefficient for the independent variable (mindfulness) in the third step is less than the result of the first step, this represents the mediating effect is partial. Complete mediation exists when mindfulness has no effect on social engagement when stigma is controlled. Statistical significance was set at 0.05. Across all regressions, we controlled for BMI, employment status, education level, personal monthly income, and monthly per capita household income.

\section{Results}

\section{Preliminary analysis}

The participants were 937 Chinese BCSs (Table 1). Most participants were aged over 56 years. A total of 614 participants with normal BMI; and 807 participants were married. Most participants were educated in high school (45.1\%) and below high school (38.4\%). Only a few participants $(4.80 \%)$ were still working. Among the participants who underwent surgery, 526 had a radical mastectomy. Most participants had more than 5 years since the time of diagnosis, and most had comorbidity. Social engagement levels differed significantly by participant BMI $(F=4.10, P=0.007)$, education level $(F=14.82, P<0.001)$, employment status $(F=5.10$, $P=0.006)$, personal monthly income $(F=10.48, P<0.001)$, and monthly per capita household income $(F=9.43$, $P<0.001)$.

\section{Reliability of measures and mean scores}

The mean scores of mindfulness, stigma, and social engagement are shown in Table 2. The Cronbach's alpha values for the MAAS (0.89), SSCI-8 (0.90), and ISE (0.84) were strong.

\section{Correlation analysis}

Mindfulness and stigma were inversely related $(r=-0.51$, $P<0.001)$. Stigma and social engagement were inversely related ( $r=-0.17, P<0.001$; Table 2). Mindfulness and social engagement were positively related $(r=0.12$, $P<0.001)$.

\section{Mediation analysis}

In Fig. 1, results suggested that stigma completely mediates the effect of mindfulness on social engagement in BCSs. Because participants' BMI, employment status, education level, personal monthly income, and monthly per capita household income could affect social engagement, they were treated as covariates. This means that at each step of the regression equation, these variables are added to the model. In the first regression equation, mindfulness positively influenced social engagement $(\beta=0.118, P<0.001)$. In the second regression equation, mindfulness negatively influenced stigma $(\beta=-0.424, P<0.001)$. In the third regression equation, stigma negatively influenced social engagement $(\beta=-0.150, P<0.001)$, while mindfulness had no significant positive influence $(\beta=0.054, P=0.113)$.

\section{Discussion}

Results indicate that mindfulness was positively correlated with social engagement, and stigma was negatively correlated with mindfulness and social engagement among Chinese BCSs. Stigma is a complete mediator in the relationship between mindfulness and social engagement in BCSs.

Social engagement levels of BCSs were significantly different in BMI, education level, employment status, personal monthly income, and monthly per capita household income. The higher the BMI, the less participation in leisure and social activities, which is consistent with other studies [42]. Compared with the general population, bias and discrimination associated with obesity contribute to diminished social engagement for the obese [43]. Education is not only related to social engagement but also considered to be one of the strongest predictors of participation in social activities [44]. More educated BCSs have more extensive information, have more opportunities and time to participate in organized social and leisure activities provided by communities and clubs, and have a higher overall quality of life. Employment status was considered to be one of the elements of social engagement [45]. Most participants in our study were retired or unemployed. The economic situation of the unemployed and retired may be poor, which leads to the correlation between social engagement and employment status. In addition, prior studies have suggested that unemployed individuals restricted their own social engagement [46]. Some studies reported that an individual's socioeconomic status was related to social engagement [47, 48]. BCSs with better socioeconomic status have more resources to maintain social connections and participate in social activities. Moreover, 
Table 1 Differences among sample characteristics and social engagement

\begin{tabular}{|c|c|c|c|c|c|}
\hline Characteristics & $N$ & $\%$ & Mean \pm SD & $t / F$ & $P$ \\
\hline Age(years) & & & & 2.49 & 0.083 \\
\hline$\leq 55$ & 188 & 20.06 & $3.20 \pm 0.71$ & & \\
\hline $56-65$ & 501 & 53.47 & $3.21 \pm 0.63$ & & \\
\hline$\geq 66$ & 248 & 26.47 & $3.31 \pm 0.63$ & & \\
\hline BMI $\left(\mathrm{kg} / \mathrm{m}^{2}\right)$ & & & & 4.10 & 0.007 \\
\hline Underweight $(<18.5)$ & 19 & 2.03 & $3.27 \pm 0.64$ & & \\
\hline Normal weight(18.5-24.9) & 614 & 65.53 & $3.28 \pm 0.64$ & & \\
\hline Overweight(25.0-29.9) & 218 & 23.27 & $3.10 \pm 0.61$ & & \\
\hline Obese $(\geq 30)$ & 86 & 9.18 & $3.22 \pm 0.71$ & & \\
\hline Marital status & & & & 1.43 & 0.154 \\
\hline Married & 807 & 86.13 & $3.24 \pm 0.65$ & & \\
\hline Unmarried/widowed/divorced & 130 & 13.87 & $3.16 \pm 0.63$ & & \\
\hline Education level & & & & 14.82 & $<0.001$ \\
\hline$<$ High school & 360 & 38.42 & $3.10 \pm 0.65$ & & \\
\hline High school & 423 & 45.14 & $3.28 \pm 0.62$ & & \\
\hline$>$ High school & 154 & 16.44 & $3.40 \pm 0.66$ & & \\
\hline Employment status & & & & 5.10 & 0.006 \\
\hline Employed & 45 & 4.80 & $3.36 \pm 0.83$ & & \\
\hline Unemployed & 105 & 11.20 & $3.06 \pm 0.71$ & & \\
\hline Retired & 787 & 83.99 & $3.25 \pm 0.62$ & & \\
\hline Personal monthly income (CNY¥) & & & & 10.48 & $<0.001$ \\
\hline$\leq 3000$ & 264 & 28.18 & $3.09 \pm 0.66$ & & \\
\hline $3001-6000$ & 601 & 64.14 & $3.28 \pm 0.64$ & & \\
\hline$\geq 6001$ & 72 & 7.68 & $3.40 \pm 0.61$ & & \\
\hline $\begin{array}{l}\text { Monthly per capita household income } \\
\text { (CNY¥) }\end{array}$ & & & & 9.43 & $<0.001$ \\
\hline$\leq 3000$ & 116 & 12.38 & $3.09 \pm 0.69$ & & \\
\hline $3001-6000$ & 551 & 58.80 & $3.20 \pm 0.65$ & & \\
\hline$\geq 6001$ & 270 & 28.82 & $3.36 \pm 0.61$ & & \\
\hline Time since diagnosis (years) & & & & 1.08 & 0.357 \\
\hline$\leq 1$ & 19 & 2.03 & $3.11 \pm 0.61$ & & \\
\hline $1-3$ & 104 & 11.10 & $3.14 \pm 0.75$ & & \\
\hline $3-5$ & 154 & 16.44 & $3.22 \pm 0.61$ & & \\
\hline$>5$ & 660 & 70.44 & $3.25 \pm 0.64$ & & \\
\hline Surgery type & & & & 2.21 & 0.085 \\
\hline Radical mastectomy & 526 & 56.14 & $3.26 \pm 0.65$ & & \\
\hline Modified radical mastectomy & 79 & 8.43 & $3.34 \pm 0.70$ & & \\
\hline Simple mastectomy & 236 & 25.19 & $3.17 \pm 0.64$ & & \\
\hline Partial mastectomy & 92 & 9.82 & $3.15 \pm 0.62$ & & \\
\hline Comorbidity & & & & 1.36 & 0.174 \\
\hline Yes & 755 & 80.58 & $3.17 \pm 0.64$ & & \\
\hline No & 182 & 19.42 & $3.25 \pm 0.65$ & & \\
\hline
\end{tabular}

All incomes are presented in Chinese yuan

$N$, number; $S D$, standard deviation their burden on disease treatment and living expenses is relatively lighter, so there are fewer negative emotions and a better level of social participation.

Our study indicates that mindfulness not just directly affected the social engagement of BCSs, it also indirectly affected their social engagement through stigma. The positive correlation between mindfulness and social engagement is consistent with existing research results [31]. Studies suggested that mindfulness can improve the level of engagement by helping individuals to view social activities in a new and 
Table 2 Correlation between mindfulness, stigma and social engagement

\begin{tabular}{llllccc}
\hline Scale & Mean & SD & $\begin{array}{l}\text { Cronbach's } \\
\text { alpha }\end{array}$ & Mindfulness & Stigma & $\begin{array}{l}\text { Social } \\
\text { engage- } \\
\text { ment }\end{array}$ \\
\hline Mindfulness & 4.60 & 0.71 & 0.89 & 1.00 & \\
Stigma & 1.62 & 0.59 & 0.90 & $-0.51^{* * * *}$ & 1.00 & \\
Social engagement & 3.23 & 0.65 & 0.84 & $0.12^{* * *}$ & $-0.17^{* * *}$ & 1.00 \\
\hline
\end{tabular}

$S D$, standard deviation

${ }^{*} P<0.05,{ }^{* *} P<0.01,{ }^{* * * *} P<0.001$

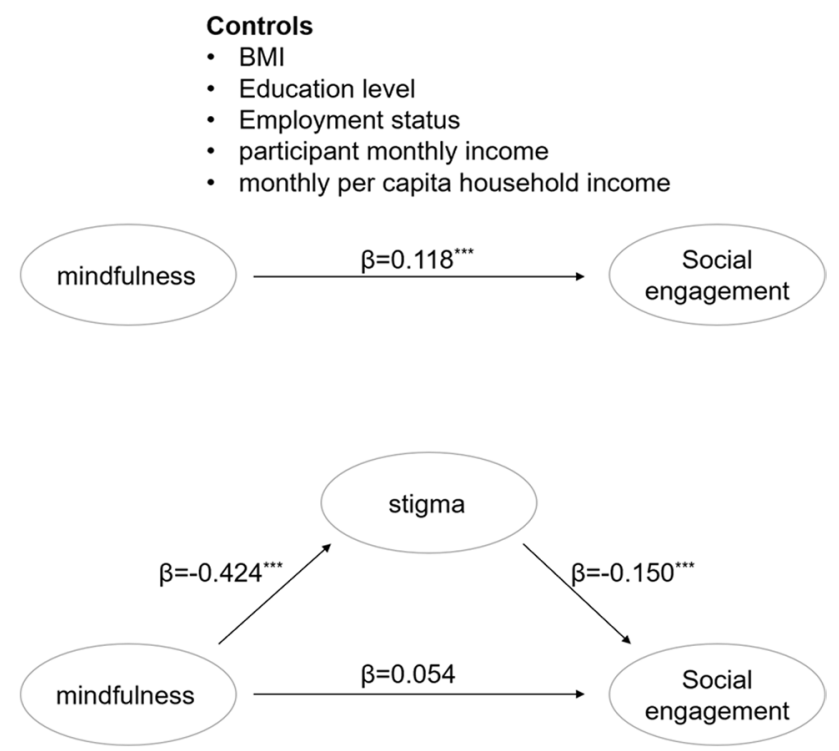

Fig. 1 Coefficients for total, direct, and indirect effects of mindfulness on social engagement. $* P<0.05, * * P<0.01$, and $* * * P<0.001$

interesting way [49]. In addition, there is a negative relationship between mindfulness and stigma in our study. Mindfulness can reduce excessive negative emotional responses to discriminatory stereotypes and thereby reduce the level of perceived stigma [50]. And for internalized stigma, mindfulness can reduce its level by increasing BCSs' acceptance of the illness and symptoms and reducing their negative self-evaluation [28]. Baron and Kenny [41] emphasize that when the independent variable has no effect on the dependent variable, and the mediator variable is controlled, their complete mediation exists. For Chinese BCSs, the stigma in this study is a complete mediator of the relationship between mindfulness and social engagement, given that this relationship becomes insignificant when the stigma is controlled. This result suggests that health care providers should identify and eliminate the constraints, which restrain the reduction of stigma level, while individual mindfulness is being enhanced in the practice of individual mindfulness intervention on social engagement of BCSs.
At present, mindfulness-based interventions such as mindfulness-based stress reduction (MBSR) and mindfulness-based cognitive therapy (MBCT) have been widely used in the treatment of physical and psychosocial problems of BCSs [51-53]. MBCT not only reduces the negative psychology (including anxiety and depression) of Chinese BCSs but also improves their positive attitude toward daily life and greatly improves their mental health [54]. Additionally, MBSR can increase interpersonal skills to promote engagement in social activities [31].

In China, the stigma of BCSs is related to changes in body image and support from family and friends [21]. Therefore, encouraging BCSs to accept changes in body image, increasing social support, helping them learn more knowledge about breast cancer, and teaching them to adopt more positive coping strategies may be effective measures to reduce the level of stigma. Subsequent studies can try to further explore the current status of the stigma of breast cancer survivors and their intervention measures.

There are some limitations to this study. First, the samples in this study were all from the SCRC. Since the club regularly organized various cancer rehabilitation activities, it might affect the levels of mindfulness, stigma, and social engagement of BCSs. Second, this study used an online survey published through WeChat. On the one side, the purpose and significance of the study could not be further elaborated to the respondents, resulting in a relatively low participation rate. In addition, it was impossible to explain the contents of the questionnaire face-to-face, which may lead to bias in content understanding. However, considering that the online investigation was more convenient to organize and conduct during the pandemic of coronavirus disease 2019 (COVID19), it had its unique advantages and ensured the smooth conduct of the study. Besides, the use of the online questionnaire meant that there might be behaviors such as filling in without reading and repeated submissions, which threaten the quality of the study. In this regard, we limited that people using the same device or the same WeChat account to fill out the questionnaire only once through the background setting, which effectively prevented duplicate submissions. However, people who had multiple WeChat accounts and devices could bypass this restriction. At the same time, logic 
tests were designed in the questionnaire to prevent people from filling in without reading the questions. Third, a crosssectional study cannot explain the causal direction of effects between variables, and therefore the interpretation of certain results should be cautious. A study proposed a delineation of mediational effects derived from cross-sectional designs into the terms temporal and atemporal associations to emphasize time in conceptualizing process models [55]. In addition, there is evidence to support the mediation mechanism that individual mindfulness affects behavior by influencing emotions [32, 33]. All in all, the current cross-sectional study can provide initial information as a basis for further longitudinal or experimental research that can confirm the conclusions of this study. Finally, only stigma as a mediator variable was included to explore the relationship between mindfulness and social engagement. Other factors may also have effects on the relationship in BCSs, such as self-esteem, optimism, and self-efficacy.

\section{Conclusion}

This study indicates that stigma plays a complete mediator role in the relationship between mindfulness and social engagement in BCSs. This result suggests that in the practice of individual mindfulness intervention on social engagement of BCSs, health care providers should identify and eliminate the constraints, which restrain the reduction of stigma level while individual mindfulness is being enhanced.

Acknowledgements We are very grateful to the Shanghai Cancer Rehabilitation Club for supporting this study. We are grateful to all the breast cancer survivors, workers, volunteers, and Hayes who provided the PROCESS macro for SPSS.

Author contribution N.J. analyzed the data and wrote the first draft of the manuscript. J.Z. and Y.X.Z. participated in the revision of the manuscript. H.Y.S. and T.W. participated in data collection and analysis. J.M.Y. participated in the design of the study and made many suggestions for the analysis and interpretation of the data. W.J. and J.W.W. were the project coordinators and participated in all parts of the work. All authors read and made critical revisions to the paper and approved the final manuscript.

Funding This study was supported by the Discipline Talents Construction Plan of Shanghai Three-year Action Plan for Public Health System Construction (2020-2022).

Data availability The datasets used to support the findings of this study are available from the corresponding author on reasonable request.

Code availability Not applicable.

\section{Declarations}

Ethics approval This study was approved by the Medical Research Ethics Committee of the School of Public Health, Fudan University (the international registry no. IRB00002408 and FWA00002399).

Consent to participate Informed consent was obtained from all individual participants included in the study.

Consent for publication Not applicable.

Competing interests The authors declare no competing interests.

\section{References}

1. Sung H, Ferlay J, Siegel RL, Laversanne M, Soerjomataram I, Jemal A, Bray F (2021) Global cancer statistics 2020: GLOBOCAN estimates of incidence and mortality worldwide for 36 cancers in 185 countries. CA Cancer J Clin 71:209-249.

2. Bodai BI, Tuso P (2015) Breast cancer survivorship: a comprehensive review of long-term medical issues and lifestyle recommendations. Permanente J 19:48-79. https://doi.org/10.7812/ tpp/14-241

3. Miovic M, Block S (2007) Psychiatric disorders in advanced cancer. Cancer 110:1665-1676

4. Vendrusculo Fangel LM, Panobianco MS, Kebbe LM, de Almeida AM, Gozzo TdO (2013) Qualify of life and daily activities performance after breast cancer treatment. Acta Paulista De Enfermagem 26:93-100. https://doi.org/10.1590/s0103-21002 013000100015

5. Bassuk SS, Glass TA, Berkman LF (1999) Social disengagement and incident cognitive decline in community-dwelling elderly persons. Ann Intern Med 131:165-173. https://doi.org/10.7326/ 0003-4819-131-3-199908030-00002

6. Curvers N, Pavlova M, Hajema K, Groot W, Angeli F (2018) Social participation among older adults $(55+)$ : results of a survey in the region of South Limburg in the Netherlands. Health Soc Care Community 26:e85-e93

7. Petruseviciene D, Surmaitiene D, Baltaduoniene D, Lendraitiene E (2018) Effect of community-based occupational therapy on health-related quality of life and engagement in meaningful activities of women with breast cancer. Occup Ther Int 2018:6798697. https://doi.org/10.1155/2018/6798697

8. Keesing S, Rosenwax L, McNamara B (2018) The implications of women's activity limitations and role disruptions during breast cancer survivorship. Women's Health 14:1745505718756381

9. Cooper CL, Phillips LH, Johnston M, Whyte M, MacLeod MJ (2015) The role of emotion regulation on social participation following stroke. Br J Clin Psychol 54:181-199

10. Kim H-M, HWA OM (2019) Relationships among self-efficacy, social support, and community participation in breast cancer survivors. Korean Journal of Occupational Therapy 27:69-82.

11. Wagner GJ, Aunon FM, Kaplan RL, Karam R, Khouri D, Tohme J, Mokhbat J (2013) Sexual stigma, psychological well-being and social engagement among men who have sex with men in Beirut, Lebanon. Cult Health Sex 15:570-582

12. Corrigan PW, Watson AC, Barr L (2006) The self-stigma of mental illness: implications for self-esteem and self-efficacy. J Soc Clin Psychol 25:875-884

13. Corrigan PW, Watson AC (2002) The paradox of self-stigma and mental illness. Clin Psychol Sci Pract 9:35 
14. Major B, O'brien LT (2005) The social psychology of stigma. Annu. Rev. Psychol. 56:393-421

15. Segalovich J, Doron A, Behrbalk P, Kurs R, Romem P (2013) Internalization of stigma and self-esteem as it affects the capacity for intimacy among patients with schizophrenia. Arch Psychiatr Nurs 27:231-234

16. Chan KK, Mak WW (2014) The mediating role of self-stigma and unmet needs on the recovery of people with schizophrenia living in the community. Qual Life Res 23:2559-2568

17. Hasan Shiri F, Mohtashami J, Nasiri M, Manoochehri H, Rohani C (2018) Stigma and related factors in Iranian people with cancer. Asian Pac J Cancer Prev 19:2285-2290. https://doi.org/10. 22034/APJCP.2018.19.8.2285.

18. MacDonald LD, Anderson HR (1984) Stigma in patients with rectal cancer: a community study. J Epidemiol Community Health 38:284-290. https://doi.org/10.1136/jech.38.4.284

19. Meacham E, Orem J, Nakigudde G, Zujewski JA, Rao D (2016) Exploring stigma as a barrier to cancer service engagement with breast cancer survivors in Kampala, Uganda. Psychooncology 25:1206-1211. https://doi.org/10.1002/pon.4215

20. Nakash O, Granek L, Cohen M, David MB (2020) Association between cancer stigma, pain and quality of life in breast cancer. Psychology, Community \& Health 8:275-287

21. Jin R, Xie T, Zhang L, Gong N, Zhang J (2021) Stigma and its influencing factors among breast cancer survivors in China: a cross-sectional study. European Journal of Oncology Nursing 52:101972

22. Pettigrew TF, Tropp LR (2006) A meta-analytic test of intergroup contact theory. J Pers Soc Psychol 90:751

23. Tang Q, Yang S, Liu C, Li L, Chen X, Wu F, Huang X (2021) Effects of mindfulness-based cognitive therapy on stigma in female patients with schizophrenia. Front Psych 12:1226

24. Wong CCY, Knee CR, Neighbors C, Zvolensky MJ (2019) Hacking stigma by loving yourself: a mediated-moderation model of self-compassion and stigma. Mindfulness 10:415-433. https://doi. org/10.1007/s12671-018-0984-2

25. Hughto JMW, Reisner SL, Pachankis JE (2015) Transgender stigma and health: a critical review of stigma determinants, mechanisms, and interventions. Soc Sci Med 147:222-231. https://doi. org/10.1016/j.socscimed.2015.11.010

26. Kabat-Zinn J (2009) Wherever you go, there you are: mindfulness meditation in everyday life. Hachette Books

27. Chan KKS, Leung DCK (2021) The impact of mindfulness on self-stigma and affective symptoms among sexual minorities. J Affect Disord 286:213-219

28. Yilmaz E, Kavak F (2020) Effects of mindfulness-based psychoeducation on the internalized stigmatization level of patients with schizophrenia. Clin Nurs Res 29:496-503. https://doi.org/ $10.1177 / 1054773818797871$

29. Chan KKS, Lee CW, Mak WW (2018) Mindfulness model of stigma resistance among individuals with psychiatric disorders. Mindfulness 9:1433-1442

30. Yang X, Mak WW (2017) The differential moderating roles of self-compassion and mindfulness in self-stigma and well-being among people living with mental illness or HIV. Mindfulness 8:595-602

31. Wells RE, Kerr C, Dossett ML, Danhauer SC, Sohl SJ, Sachs BC, Feeley JW, Wolkin J, Wall R, Kaptchuk T (2019) Can adults with mild cognitive impairment build cognitive reserve and learn mindfulness meditation? Qualitative theme analyses from a small pilot study. J Alzheimers Dis 70:825-842

32. Maynard BR, Solis MR, Miller VL, Brendel KE (2017) Mindfulness-based interventions for improving cognition, academic achievement, behavior, and socioemotional functioning of primary and secondary school students. Campbell Syst Rev 13:1-144
33. Malinowski P, Lim HJ (2015) Mindfulness at work: positive affect, hope, and optimism mediate the relationship between dispositional mindfulness, work engagement, and well-being. Mindfulness 6:1250-1262. https://doi.org/10.1007/s12671-015-0388-5

34. Tacón AM, Caldera YM, Ronaghan C (2004) Mindfulness-based stress reduction in women with breast cancer. Fam Syst Health 22:193

35. Lopes PN, Salovey P, Côté S, Beers M, Petty RE (2005) Emotion regulation abilities and the quality of social interaction. Emotion $5: 113$

36. Schoemann AM, Boulton AJ, Short SD (2017) Determining power and sample size for simple and complex mediation models. Soc Psychol Pers Sci 8:379-386. https://doi.org/10.1177/1948550617 715068

37. Brown KW, Ryan RM (2003) The benefits of being present: mindfulness and its role in psychological well-being. J Pers Soc Psychol 84:822-848. https://doi.org/10.1037/0022-3514.84.4.822

38. Deng YQ, Li S, Tang YY, Zhu LH, Ryan R, Brown K (2012) Psychometric properties of the Chinese translation of the Mindful Attention Awareness Scale (MAAS). Mindfulness 3:10-14. https://doi.org/10.1007/s12671-011-0074-1

39. Molina Y, Choi SW, Cella D, Rao D (2013) The Stigma Scale for Chronic Illnesses 8-item version (SSCI-8): development, validation and use across neurological conditions. Int J Behav Med 20:450-460. https://doi.org/10.1007/s12529-012-9243-4

40. Mor V, Branco K, Fleishman J, Hawes C, Phillips C, Morris J, Fries B (1995) The structure of social engagement among nursinghome residents. J Gerontol B-Psychol 50:P1-P8. https://doi.org/ 10.1093/geronb/50B.1.P1

41. Baron RM, Kenny DA (1986) The moderator mediator variable distinction in social psychological-research - conceptual, strategic, and statistical considerations. J Pers Soc Psychol 51:1173-1182. https://doi.org/10.1037/0022-3514.51.6.1173

42. Howe LD, Kanayalal R, Harrison S, Beaumont RN, Davies AR, Frayling TM, Davies NM, Hughes A, Jones SE, Sassi F, Wood AR, Tyrrell J (2020) Effects of body mass index on relationship status, social contact and socio-economic position: Mendelian randomization and within-sibling study in UK Biobank. Int J Epidemiol 49:1173-1184. https://doi.org/10.1093/ije/dyz240

43. Puhl R, Brownell KD (2001) Bias, discrimination, and obesity. Obes Res 9:788-805. https://doi.org/10.1038/oby.2001.108

44. Rodríguez-Galán MB, Falcón LM (2010) Patterns of social activity engagement among older Hispanics and their relationship to sociodemographic and health variables. Act Adapt Aging $34: 251-275$

45. Shibayama T, Noguchi H, Takahashi H, Tamiya N (2018) Relationship between social engagement and diabetes incidence in a middle-aged population: results from a longitudinal nationwide survey in Japan. J Diabetes Invest 9:1060-1066. https://doi.org/ 10.1111/jdi.12820

46. Meek KP, Bergeron CD, Towne SD, Ahn S, Ory MG, Smith ML (2018) Restricted social engagement among adults living with chronic conditions. Int J Env Res Pub He 15. https://doi.org/10. 3390/ijerph15010158.

47. Asadollahi A, Saberi LF, Tabrizi AM, Faraji N (2011) Do public health and social participation matter for the elderly? An analysis of an aging community in Khuzistan Province. Iran Canadian Social Science 7:240-244

48. Serrat R, Villar F, Celdrán M (2015) Factors associated with Spanish older people's membership in political organizations: the role of active aging activities. Eur J Ageing 12:239-247

49. Leroy H, Anseel F, Dimitrova NG, Sels L (2013) Mindfulness, authentic functioning, and work engagement: a growth modeling approach. J Vocat Behav 82:238-247. https://doi.org/10.1016/j. jvb.2013.01.012 
50. Gamwell KL, Roberts CM, Espeleta HC, Baudino MN, Hommel KA, Grunow JE, Jacobs NJ, Gillaspy SR, Mullins LL, Chaney JM (2020) Perceived stigma, illness uncertainty, and depressive symptoms in youth with inflammatory bowel disease: the moderating effect of mindfulness. Psychol Health Med 25:1037-1048. https://doi.org/10.1080/13548506.2020.1714062

51. Shapiro SL, Bootzin RR, Figueredo AJ, Lopez AM, Schwartz GE (2003) The efficacy of mindfulness-based stress reduction in the treatment of sleep disturbance in women with breast cancer - an exploratory study. J Psychosom Res 54:85-91. https://doi.org/10. 1016/S0022-3999(02)00546-9

52. Zhang JY, Zhou YQ, Feng ZW, Fan YN, Zeng GC, Wei L (2017) Randomized controlled trial of mindfulness-based stress reduction (MBSR) on posttraumatic growth of Chinese breast cancer survivors. Psychol Health Med 22:94-109. https://doi.org/10.1080/ 13548506.2016.1146405

53. Johannsen M, O'Connor M, O'Toole MS, Jensen AB, Hojris I, Zachariae R (2016) Efficacy of mindfulness-based cognitive therapy on late post-treatment pain in women treated for primary breast cancer: a randomized controlled trial. J Clin Oncol 34:3390-3399. https://doi.org/10.1200/JCO.2015.65.0770

54. Chu XM, Lou Y, Wang ZJ, Xu Q (2020) Effect of mindfulness based cognitive therapy on the mental health and quality of life in patients with breast cancer. Indian J Pharm Sci 82:48-52. https:// doi.org/10.36468/pharmaceutical-sciences.spl.143.

55. Winer ES, Cervone D, Bryant J, McKinney C, Liu RT, Nadorff MR (2016) Distinguishing mediational models and analyses in clinical psychology: atemporal associations do not imply causation. J Clin Psychol 72:947-955

Publisher's note Springer Nature remains neutral with regard to jurisdictional claims in published maps and institutional affiliations. 